\title{
Long-term outcomes of preoperative docetaxel with cisplatin plus S-1 therapy for gastric cancer with extensive nodal metastasis (JCOG1002)
}

\author{
Daisuke Takahari ${ }^{1}$ (D) Seiji Ito ${ }^{2} \cdot$ Junki Mizusawa $^{3} \cdot$ Hiroshi Katayama $^{3} \cdot$ Masanori Terashima $^{4} \cdot$ Mitsuru Sasako $^{5}$. \\ Shinji Morita ${ }^{6} \cdot$ Takashi Nomura $^{7} \cdot$ Makoto Yamada $^{8} \cdot$ Yoshiyuki Fujiwara $^{9} \cdot$ Yutaka Kimura $^{10}$ - Atsuki Ikeda ${ }^{11}$. \\ Yoshio Kadokawa ${ }^{12}$. Takeshi Sano ${ }^{13}$ on behalf of the Stomach Cancer Study Group of the Japan Clinical Oncology \\ Group
}

Received: 10 June 2019 / Accepted: 2 September 2019 / Published online: 12 September 2019

(C) The International Gastric Cancer Association and The Japanese Gastric Cancer Association 2019

\begin{abstract}
Background Preoperative chemotherapy with cisplatin plus S-1 (CS) followed by gastrectomy with D2 plus para-aortic lymph node (PAN) dissection is regarded as a standard treatment in Japan for advanced gastric cancer with bulky lymph node (BN) and/or PAN metastasis. In the JCOG1002, we added docetaxel to CS (DCS) to further improve long-term outcomes. However, the primary endpoint, clinical response rate (RR), did not reach the expected level (Ito et al. in Gastric Cancer 20:322-31, 2017). Herein, we report our long-term survival results.

Methods Patients with BN and/or PAN metastasis received 2 or 3 cycles of DCS therapy (docetaxel at $40 \mathrm{mg} / \mathrm{m}^{2}$ and cisplatin at $60 \mathrm{mg} / \mathrm{m}^{2}$ on day 1 and S-1 at $80 \mathrm{mg} / \mathrm{m}^{2}$ per day for 2 weeks, followed by a 2-week rest) followed by gastrectomy with D2 plus PAN dissection and postoperative S-1 for 1 year.

Results Between July 2011 and May 2013, 53 patients were enrolled. Clinically, 17.0\% had both PAN and BN metastasis, and the rest had either PAN (26.4\%) or BN (56.6\%) metastasis. Among all eligible patients, the 5-year overall survival was 54.9\% (95\% confidence interval 40.3-67.3\%) at the last follow-up in May 2018. Among 44 eligible patients with R0 resection, the 5-year relapse-free survival was $47.7 \%$ (95\% confidence interval 32.5-61.5\%).

Conclusions Adding docetaxel to CS in preoperative chemotherapy for extensive nodal metastasis improved neither shortterm outcomes nor long-term survival. Preoperative chemotherapy with CS followed by D2 + PAN dissection and postoperative S-1 remains the standard of care for patients with extensive nodal metastasis.
\end{abstract}

Keywords Gastric cancer $\cdot$ Extensive lymph node metastasis $\cdot$ Preoperative chemotherapy $\cdot$ DCS

Daisuke Takahari

daisuke.takahari@jfcr.or.jp

1 Department of Gastroenterology, The Cancer Institute Hospital of Japanese Foundation for Cancer Research, 3-8-31 Ariake, Koto-ku, Tokyo 135-8550, Japan

2 Department of Gastroenterological Surgery, Aichi Cancer Center Hospital, Nagoya, Japan

3 Japan Clinical Oncology Group Data Center/Operations Office, National Cancer Center Hospital, Chuo-ku, Tokyo, Japan

4 Division of Gastric Surgery, Shizuoka Cancer Center, Shizuoka, Japan

5 Division of Upper Gastrointestinal Surgery, Department of Surgery, Hyogo College of Medicine, Nishinomiya, Japan

6 Division of Gastric Surgery, National Cancer Center Hospital, Tokyo, Japan
7 Department of Surgery, Yamagata Prefectural Central Hospital, Yamagata, Japan

8 Department of Surgery, Gifu Municipal Hospital, Gifu, Japan

9 Department of Surgery, Osaka Medical Center for Cancer and Cardiovascular Disease, Osaka, Japan

10 Department of Surgery, Sakai City Medical Center, Sakai, Japan

11 Division of Gastroenterology, Department of Internal Medicine, Kobe University School of Medicine, Kobe, Japan

12 Department of Gastroenterological Surgery, Tenri Hospital, Tenri, Japan

13 Department of Surgery, The Cancer Institute Hospital of Japanese Foundation for Cancer Research Cancer Institute Hospital, Tokyo, Japan 


\section{Introduction}

For locally advanced gastric cancer (LAGC), surgical resection is the mainstay of treatment. However, in some subgroups of patients with LAGC such as those with extensive lymph node metastasis (ELM), which is defined as para-aortic lymph node (PAN) metastasis, or bulky lymph nodes (BN) along the celiac, splenic, common hepatic, or proper hepatic arteries, or both PAN and BN, the prognosis remains poor even if curative resection is achieved [1]. According to data collected by the Stomach Cancer Study Group (SCSG) of the Japan Clinical Oncology Group (JCOG), the 3-year survival of 86 patients with both clinical and pathological PAN metastasis who underwent surgery was only $5 \%$. According to the SCSG/JCOG, several phase II studies have been conducted to evaluate the efficacy and safety of preoperative chemotherapy followed by gastrectomy with D2 plus PAN dissection for gastric cancer with ELM (JCOG0001 [2], JCOG0405 [3]). JCOG0405 was conducted to assess the effects of administering 2 or 3 cycles of cisplatin $\left(60 \mathrm{mg} / \mathrm{m}^{2}\right.$ on day 8$)$ and S-1 $\left(80 \mathrm{mg} / \mathrm{m}^{2}\right.$ per day from day 1 to day 21 followed by a 1 -week rest period) (CS) chemotherapy, followed by surgery [3]. This study showed an excellent response rate (RR) of $64.7 \%$ and a 3-year survival of $58.8 \%$, with no treatment-related deaths and good feasibility. Based on this result, the SCSG/JCOG have tentatively regarded preoperative CS chemotherapy as the current standard treatment for gastric cancer with ELM. As the next step, triplet therapy comprising docetaxel and CS (DCS) was introduced as preoperative chemotherapy for LAGC with ELM (JCOG1002) [4].

The JCOG1002 study was a multi-institutional, phase II trial designed to evaluate the safety and efficacy of DCS as preoperative chemotherapy for gastric cancer with ELM. In the primary analysis, the clinical RR was $57.7 \%$ [30/52, 80\% confidence interval (CI) 47.9-67.1\%, one-sided $p=0.89]$ ]. Common grade 3 or grade 4 adverse events during DCS therapy were leukocytopenia (18.9\%), neutropenia (39.6\%) and hyponatremia (15.1\%). The most common grade 3 or grade 4 surgical morbidity was abdominal infection (10.2\%). The pathological RR was $50.0 \%$ (26/52) [4]. Accordingly, we concluded that while DCS therapy was feasible, it did not yield a sufficient RR. We now report the 5-year overall survival (OS) results, which showed maturity as compared to the primary analysis.

\section{Methods}

The JCOG1002 was conducted as a multi-institutional phase II trial by the SCSG/JCOG. All data were handled by the JCOG Data Center. The protocol of this study was approved by the Protocol Review Committee of the JCOG on June 14, 2011 and the institutional review board at each participating center. All enrolled patients provided fully informed written consent. The study was undertaken in accordance with the Declaration of Helsinki and the Japanese Ethical Guidelines for Clinical Studies. The JCOG Data and Safety Monitoring Committee reviewed serious adverse events and judged whether an adverse event was attributable to treatment. This trial was registered with the University Hospital Medical Information Network Clinical Trials Registry (https://www.umin.ac.jp/ctr/) as UMIN000006069. The methods of this study were previously described in detail [4]. The main eligibility criteria were an age of 20-75 years and histologically proven primary gastric adenocarcinoma with ELM. Patients who were suitable candidates for chemotherapy and surgery were enrolled. ELM was defined as PAN metastasis (no. $16 \mathrm{a} 2 / 16 \mathrm{~b} 1$ ) or BN (one node larger than $3 \mathrm{~cm}$ or two larger than $1.5 \mathrm{~cm}$ ) along the celiac, splenic, common hepatic, or proper hepatic arteries, or both. The absence of peritoneal dissemination was confirmed by staging laparoscopy before entry into the study. An Eastern Cooperative Oncology Group performance status of 0 or 1 and no previous chemotherapy or radiotherapy were also among the requirements.

\section{Treatment}

The treatment consisted of the following three parts. First, preoperative chemotherapy was administered. Patients received an infusion of docetaxel $\left(40 \mathrm{mg} / \mathrm{m}^{2}\right)$ and cisplatin $\left(60 \mathrm{mg} / \mathrm{m}^{2}\right)$ on day 1 , and orally took $\mathrm{S}-1\left(80 \mathrm{mg} / \mathrm{m}^{2}\right.$ per day [80-120 mg/day total dose depending on the patient's body surface area: $80 \mathrm{mg}$ if $<1.25 \mathrm{~m}^{2} ; 100 \mathrm{mg}$ if $1.25-1.5 \mathrm{~m}^{2}$; and $120 \mathrm{mg}$ if $>1.5 \mathrm{~m}^{2}$ ]) for 2 weeks on days $1-14$, followed by a 2 -week rest. Two cycles of preoperative chemotherapy were administered unless obvious progression or unacceptable adverse events were observed. The possibility of R0 resection was evaluated after the second cycle. If R0 resection was considered to be feasible, the patient underwent surgery within 56 days after the last administration of S-1. If $\mathrm{R} 0$ resection was considered to be difficult despite tumor shrinkage after the second cycle, a third cycle of DCS therapy could be added before surgery. If curative resection was regarded as being difficult with no shrinkage of metastases, the protocol treatment was terminated. 
Second, surgery was performed. After laparotomy, resectability was re-evaluated, and intraperitoneal washing cytology specimens were examined. If the cytology findings were negative, $\mathrm{R} 0$ resection was attempted by $\mathrm{D} 2$ gastrectomy with PAN dissection. If R0 resection was considered to be impossible, the protocol treatment was terminated.

Third, adjuvant chemotherapy was administered. S-1 therapy was to be started within 42 days of surgery if patients had undergone R0 resection pathologically. A 6 -week cycle consisting of 4 weeks of oral administration of S-1 $80 \mathrm{mg} / \mathrm{m}^{2}$ per day $(80-120 \mathrm{mg} /$ day total dose depending on the patient's body surface area and creatinine clearance [Ccr]: $80 \mathrm{mg}$ if $<1.25 \mathrm{~m}^{2} ; 100 \mathrm{mg}$ if $1.25-1.5 \mathrm{~m}^{2}$; and $120 \mathrm{mg}$ if $>1.5 \mathrm{~m}^{2}$, and -1 level of $\mathrm{S}-1$ if $40 \leq \mathrm{Ccr}<60 \mathrm{ml} / \mathrm{min}$, protocol treatment was terminated if $\mathrm{Ccr}<40 \mathrm{ml} / \mathrm{min}$ ) followed by 2 weeks of rest was repeated during the first year after surgery. Even after R0 resection, if the tumor had progressed between preoperative chemotherapy and surgery, and a histological examination of the resected specimen showed no chemotherapeutic effect, the protocol treatment was terminated and S-1 was not administered. If S-1 therapy was not started within 12 weeks after surgery, regardless of the reason, the protocol treatment was terminated. The protocol treatment was completed when a patient had received 2 or 3 cycles of preoperative chemotherapy, had undergone R0 resection by gastrectomy with D2 plus PAN dissection, and had received 1 year of postoperative chemotherapy. After completion of the protocol treatment, no further treatment was given until tumor recurrence. Additional details, e.g., dose modifications, have been reported previously [4].

\section{Follow-up}

All enrolled patients were to be followed up for 5 years. Efficacy and safety were to be evaluated at least every 3 months until the third year, then at least every 6 months for the last 2 years. General condition and blood test results were checked every 3 months until the third year and every 6 months for the last 2 years. Abdominal computed tomography was performed every 6 months until the third year and every year for the last 2 years. Chest X-ray and upper gastrointestinal tract endoscopy were performed each year. The attending physicians assessed responses according to the Response Evaluation Criteria in Solid Tumors (RECIST, V.1.0) [5]. Safety assessments were performed on day 1 of each cycle. Safety was also evaluated in week 2 of the first cycle for postoperative chemotherapy. Adverse events were graded according to the Common Terminology Criteria for Adverse Events (V.4.0) [6].

\section{Outcomes}

The primary endpoint was the clinical RR for all eligible patients, which was evaluated by central peer review according to RECIST, V.1.0 [5]. The secondary endpoints were 3 -year overall survival, 5-year overall survival, proportion with R0 resection, proportion of completion of surgery, proportion of completion of adjuvant chemotherapy, pathological RR, and adverse events. Overall survival was defined as the time from the date of enrollment to the date of death from any cause, and was censored on the last contact for a surviving patient. As a post-hoc analysis, relapse-free survival was analyzed. Relapse-free survival was defined as the time from the date of enrollment to the first date of relapse and/or death from any cause, and was censored on the last contact for a relapse-free surviving patient. Pathological response was evaluated and graded by the institutional pathologists according to the Japanese Classification of Gastric Carcinoma, third English edition [7]: grade 1a, viable tumor cells remain in more than two thirds of the tumorous area; grade $1 \mathrm{~b}$, more than one third but less than two thirds; grade 2, less than one third; grade 3, no viable tumor cells. In this report, pathological response was defined as grade 2 to grade 3 response.

\section{Statistical analysis}

The RR was $64.7 \%$ in the JCOG0405, and the efficacy of DCS therapy was expected to be superior to that of CS therapy in the JCOG0405. The sample size was calculated to be 50 cases, under the hypothesis that the expected RR and threshold RR were $80 \%$ and $65 \%$, respectively, with use of one-sided testing at the $10 \%$ significance level with a power of $80 \%$. For the primary analysis, collection of the primary endpoint data was stopped on May 30th, 2014. The cutoff date for this long-term analysis was May 31st, 2018. We estimated time-to-events type endpoints with the Kaplan-Meier method. Overall survival was analyzed for all eligible patients. Relapse-free survival was analyzed for eligible patients with R0 resection. All statistical analyses were performed using SAS V.9.4 software.

\section{Results}

Between July 2011 and May 2013, 53 patients in total were enrolled. The baseline characteristics were reported previously [4]. One patient was found not to have $\mathrm{BN}$ as judged by central peer review, and was therefore excluded from the primary analyses. Clinically, $17.0 \%$ of patients had both PAN and BN metastasis, and the remaining patients had either PAN (26.4\%) or BN $(56.6 \%)$ metastasis. The pathological RR was $34.6 \%$ in the 52 eligible patients. At 


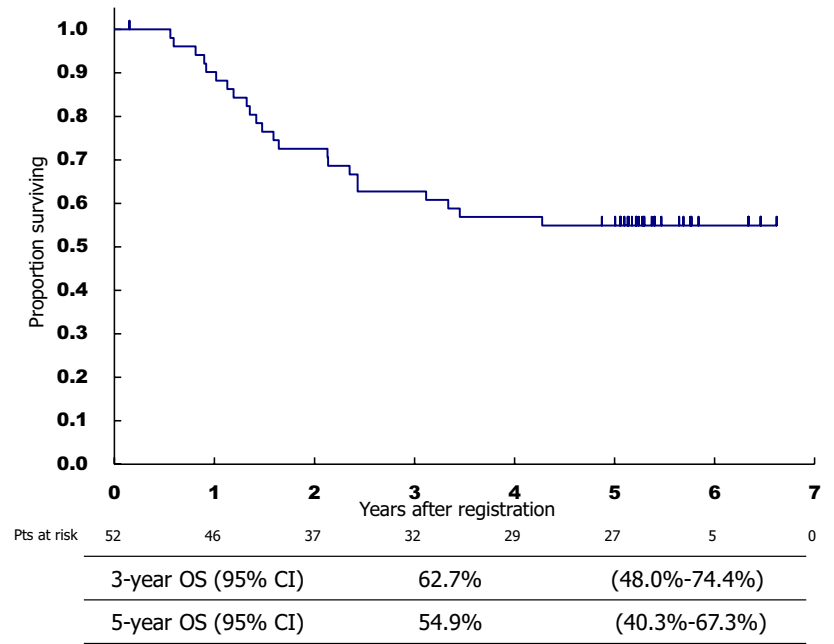

Fig. 1 Kaplan-Meier plot of overall survival. OS overall survival

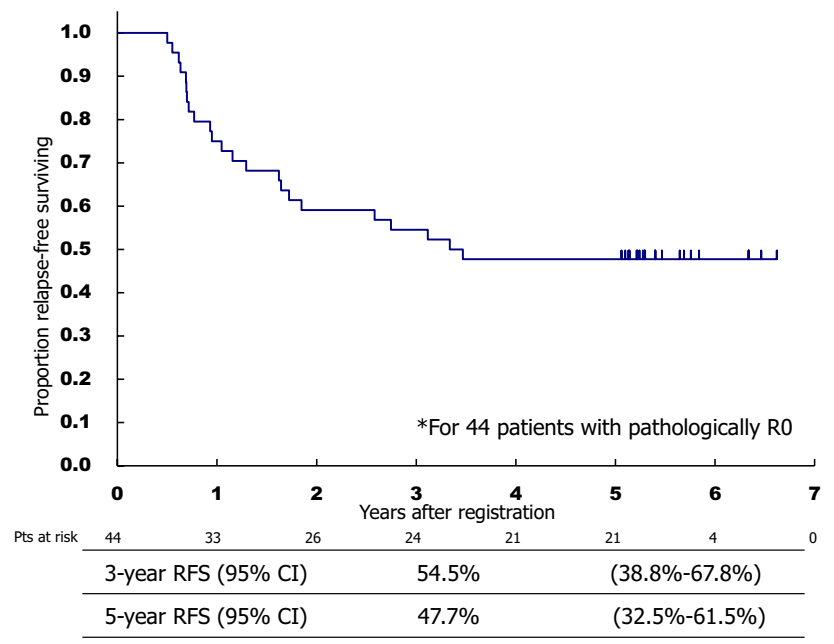

Fig. 2 Kaplan-Meier plot of relapse-free survival. RFS relapse-free survival

the cutoff date in May 2018, the median follow-up for the OS analysis was 5.0 years (range $0.2-6.6$ ). There were 23 deaths. The causes of death were progressive disease in 19 patients, other diseases in 3, and unknown in one. The 3-year OS was $62.7 \%$ (95\% CI 48.0-74.4\%) and the 5-year OS was $54.9 \%$ (95\% CI 40.3-67.3\%) (Fig. 1). Among 44 eligible patients with R0 resection, 23 recurrences were confirmed. The 3-year relapse-free survival (RFS) was 54.5\% (95\% CI 38.8-67.8\%). The 5-year RFS was $47.7 \%$ (95\% CI 32.5-61.5\%) (Fig. 2). Twenty patients developed cancer recurrence. The most frequent site of recurrence was lymph nodes $(n=10)$, followed by hematogenous $(n=8)$, peritoneum $(n=5)$, and local recurrence $(n=2)$. Subgroup analyses were conducted for groups with concomitant PAN and BN metastasis (PAN and BN metastasis; $n=9$ for OS,
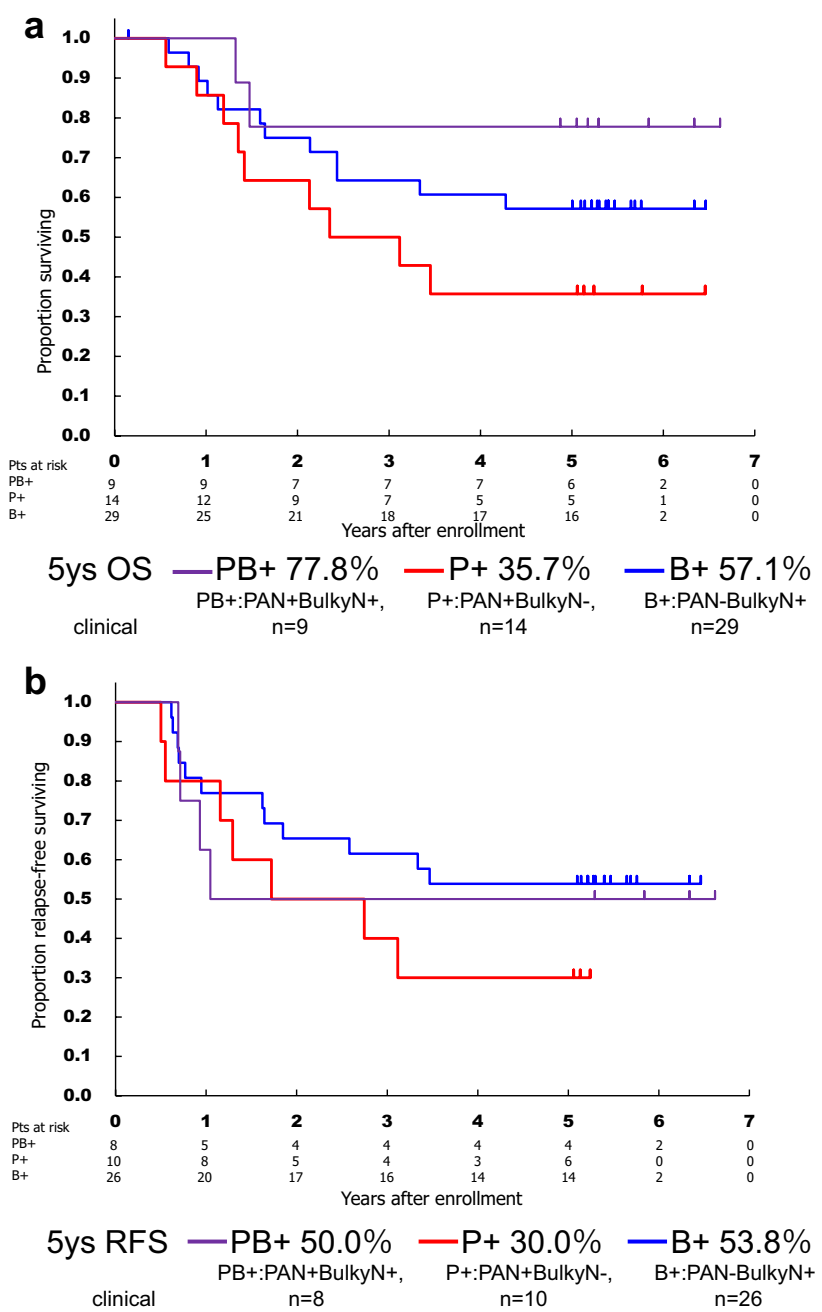

Fig. 3 a Kaplan-Meier plot of overall survival according to clinical lymph node status. b Kaplan-Meier plot of relapse-free survival according to clinical lymph node status. $P B+$ para-aortic lymph node-positive and bulky lymph node-positive, $P+$ para-aortic lymph node-positive and bulky lymph node-negative, $B+$ para-aortic lymph node-negative and bulky lymph node-positive, $5 Y$ OS 5-years overall survival, $5 Y$ RFS 5-years relapse-free survival

$n=8$ for RFS), only PAN metastasis ( $n=14$ for OS, $n=10$ for RFS) and only BN metastasis ( $n=29$ for OS, $n=26$ for RFS). The 5-year OS and 5-year RFS rates in PAN and BN metastasis were $77.8 \%$ and $50.0 \%$. The 5-year OS and 5-year RFS rates in only PAN metastasis were $35.7 \%$ and $30.0 \%$. The 5-year OS and 5-year RFS rates in only BN metastasis were $57.1 \%$ and $53.8 \%$ (Fig. 3a, b). As for pathological PAN status, the 5-year OS of the negative and positive cases was $63.2 \%$ and $42.9 \%$ and the 5-year RFS was $54.1 \%$ and not reached (Fig. 4a, b). Among the 52 eligible patients who discontinued the protocol treatment, $23(44.2 \%)$ received second-line treatment. Among them, 23 cases received chemotherapy and 3 underwent surgery, while 2 cases were managed with other approaches including duplication of 


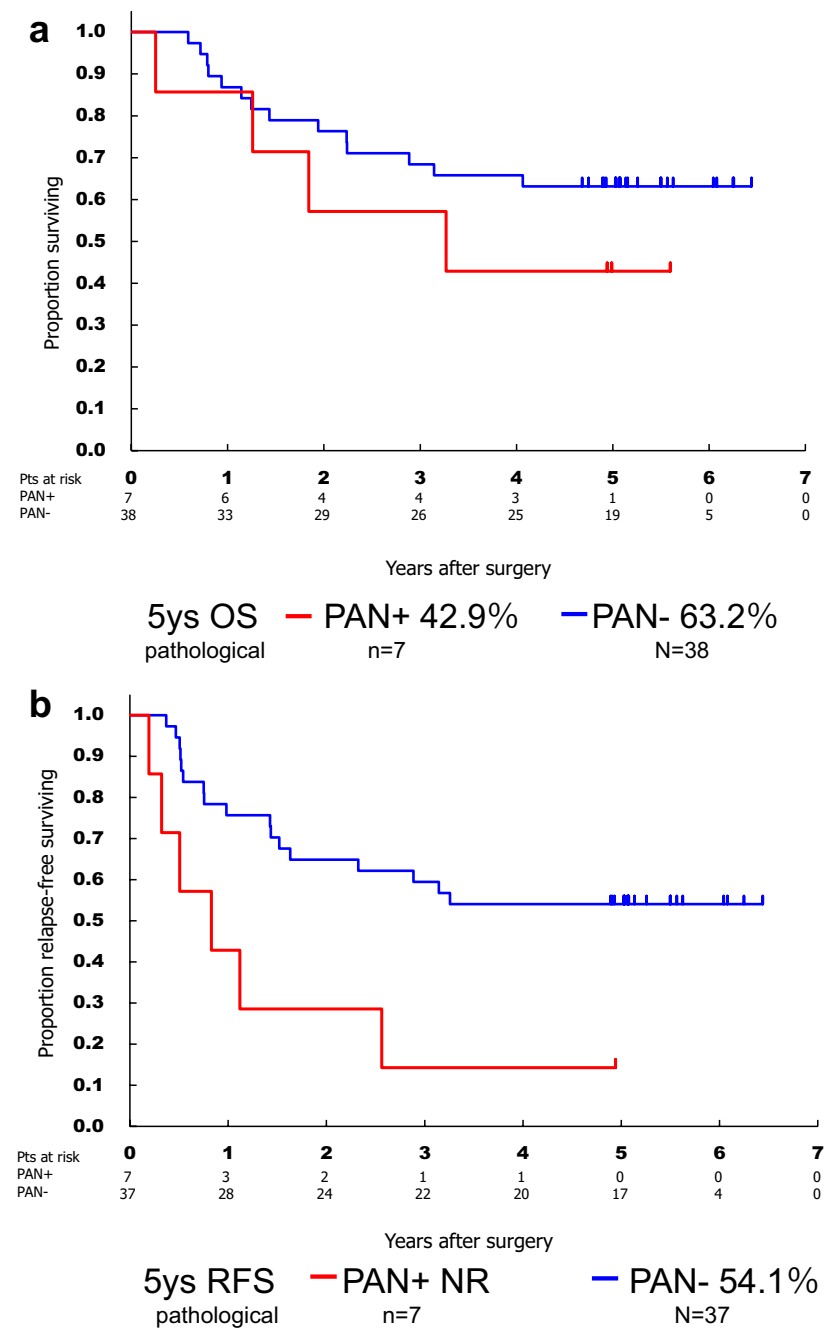

Fig. 4 a Kaplan-Meier plot of overall survival according to pathological para-aortic lymph node status. b Kaplan-Meier plot of relapsefree survival according to pathological para-aortic lymph node status. PAN+ para-aortic lymph node-positive, PAN- para-aortic lymph node-negative, $5 Y$ OS 5-years overall survival, $5 Y$ RFS 5-years relapse-free survival

prior treatments. Adverse events associated with preoperative chemotherapy and surgical complications were reported previously [4]. Grade 3 or grade 4 toxicities during postoperative chemotherapy experienced by 37 treated patients included leukopenia (5.4\%), neutropenia (24.3\%), anemia $(8.1 \%)$, thrombocytopenia $(2.7 \%)$, hypokalemia $(5.7 \%)$, increased aspartate aminotransferase $(5.4 \%)$, increased alanine aminotransferase $(5.4 \%)$, anorexia $(13.5 \%)$, diarrhea $(5.4 \%)$, nausea $(2.7 \%)$, and febrile neutropenia $(0 \%)$. There were no treatment-related deaths during protocol treatment.

\section{Discussion}

As revealed by the analysis of the secondary endpoint of OS, adding docetaxel to CS in preoperative chemotherapy for ELM did not improve long-term survival. This result is similar to that obtained in our primary analysis of this study [4]. Therefore, CS in preoperative chemotherapy with D2 plus PAN dissection followed by postoperative $\mathrm{S}-1$ remains the standard of care for LAGC with ELM.

Comparisons of three phase II studies of preoperative chemotherapy conducted by the SCSG/JCOG are summarized in Table 1. Although DCS achieved better treatment completion and tumor regression rates in cases with more than grade 2 tumors compared to the other trials with no treatment-related deaths, the 3- and 5-year OS were 63\% and $55 \%$, respectively. These results were essentially identical to those obtained with CS (the 3- and 5-year OS were $59 \%$ and $53 \%$, respectively). It is true in general that the comparison of survival outcome of different phase II trials is not appropriate. But our three phase II trials (JCOG0001 [2], JCOG0405 [3], and this trial) for this target were carried out through almost same eligibility criteria and same participating facilities. We believe that a certain level of comparability is warranted in these trials. One of the possible explanations for DCS not being superior to CS is the dose intensity of S-1 in DCS, which was lower than that of CS in the JCOG0405 (280 $\mathrm{mg} / \mathrm{m}^{2} /$ week and $420 \mathrm{mg} / \mathrm{m}^{2} /$ week, respectively). On the other hand, the incidences of grade 3 or 4 neutropenia and febrile neutropenia in the present study were lower than those reported in a phase II study of unresectable or recurrent gastric cancer [8]. The operative patients, who had favorable performance status, might have been able to tolerate more intensive chemotherapy than we had expected. Recently, the randomized phase III study JCOG1013, where

Table 1 Comparisons of three phase II studies of preoperative chemotherapy conducted by the SCSG/JCOG

\begin{tabular}{llllllllll}
\hline Trial & Regimen & $N$ & $\begin{array}{l}\text { Completion } \\
\text { rate }(\%)\end{array}$ & RR $(\%)$ & R0 $(\%)$ & $\begin{array}{l}\text { TRG }(\geq \text { Grade } \\
2)(\%)\end{array}$ & 3Y OS $(\%)$ & $5 Y$ OS $(\%)$ & TRD $(n)$ \\
\hline JCOG0001 & CDDP+IRI & 55 & 65 & 55 & 66 & 11 & 27 & 22 & 3 \\
JCOG0405 & CS & 53 & 69 & 63 & 82 & 29 & 59 & 53 & 0 \\
JCOG1002 & DCS & 53 & 81 & 58 & 85 & 35 & 63 & 55 & 0 \\
\hline
\end{tabular}

$N$ number, $R R$ response rate, $R O$ R0 resection rate, $T R G$ tumor regression grade, $O S$ overall survival, $T R D$ treatment-related death, $C D D P$ cisplatin, IRI irinotecan, $C S$ cisplatin plus $\mathrm{S}-1, D C S$ docetaxel plus cisplatin plus $\mathrm{S}-1$ 
DCS was administered to patients with unresectable gastric cancer at the same dosage as in this study, also failed to show superiority over CS (OS was 14.2 months in the DCS group, 15.3 months in the CS group; HR 0.99, 95\% CI 0.80-1.39) [9]. Taken together, these observations indicate that 2 cycles of DCS in this regimen are apparently not a promising preoperative chemotherapy for patients with gastric cancer with ELM. However, the triplet regimen may still have potential. In Europe, FLOT (5-FU/leucovorin plus oxaliplatin and docetaxel) has become a new standard preoperative chemotherapeutic regimen for LAGC [10]. A phase II study of docetaxel + oxaliplatin + S-1 (DOS) for LAGC (cT3-4N0 or cT2-4 N+) was reported from South Korea [11]. The regimen consisted of 3 cycles of DOS (docetaxel $50 \mathrm{mg} / \mathrm{m}^{2}$, oxaliplatin $100 \mathrm{mg} / \mathrm{m}^{2}$ day $1, \mathrm{~S}-180 \mathrm{mg} / \mathrm{m}^{2}$ day $1-14, \mathrm{q} 3 \mathrm{w}$; dose intensity of S $1: 373 \mathrm{mg} / \mathrm{m}^{2} /$ week) followed by surgery and S-1 for 1 year as postoperative chemotherapy. The response rate was $53.8 \%$ and the R0 resection proportion was $97.6 \%$. In addition, histological CR was confirmed in $19.5 \%$ of the patients, indicating DOS to be an excellent regimen for achieving local control. Based on these results, the phase III PRODIGY study (ClinicalTrials.gov: NCT01515748) was initiated, accrual was completed, and the results are being awaited [12]. In Japan, we have also started a trial with 3 preoperative cycles of DOS (docetaxel $40 \mathrm{mg} / \mathrm{m}^{2}$, oxaliplatin $100 \mathrm{mg} / \mathrm{m}^{2}$ day $1, \mathrm{~S}-180 \mathrm{mg} / \mathrm{m}^{2}$ day $1-14$, q3w; dose intensity of S-1: $373 \mathrm{mg} / \mathrm{m}^{2} /$ week) for LAGC (JCOG1704; jRCTs031180028), which might be more effective than the DCS in the present study through adjustment of the dose intensity of S-1.

Another explanation may involve the number of treatment cycles. In this study, most patients received 2 cycles of preoperative chemotherapy, which was specified in JCOG0001 [2] and JCOG0405 [3]. However, suitable durations of preoperative chemotherapy have not as yet been established. A randomized phase II trial to compare preoperative chemotherapy using 2 and 4 cycles of CS therapy and DCS therapy in a two-by-two factorial design for LAGC is currently being conducted [13]. In that study, 4 cycles of DCS achieved more favorable outcomes than other regimens, though the differences did not reach statistical significance. Therefore, 4 cycles of DCS may be better for preoperative chemotherapy in terms of OS, although the results of this study await confirmation.

In the present trial, there were no significant survival differences among the clinical ELM site subgroups. This is in contrast to the JCOG0405, in which survival was better in patients with only one ELM site than in those with metastatic disease involving both sites. Because there were no significant differences in the background factors of the registered cases between JCOG0405 and JCOG1002, treatments for these subgroups should be developed together, as that was the strategy of SCSG/JCOG in the past.
The RFS of patients who were pathologically positive for PAN was significantly worse than those of patients pathologically negative for PAN. Park et al. reported favorable outcomes of patients with gastric cancer with isolated PAN involvement given palliative chemotherapies, but the 3-year OS for patients with de novo PAN metastasis was only $13.1 \%$ [1]. In contrast, several patients who were pathologically positive for PAN survived for more than 5 years in the present trial. Furthermore, the pathological status cannot be determined without PAN dissection. Thus, at least for now, PAN dissection should be included in the multidisciplinary treatment strategy for this disease, although the value of PAN dissection must be carefully evaluated in future trials.

In conclusion, our updated analysis showed that adding docetaxel to CS in preoperative chemotherapy for extensive lymph node metastasis, whether PAN, BN or both, was not associated with improved long-term survival. Preoperative chemotherapy with CS followed by D2 + PAN dissection and postoperative $\mathrm{S}-1$ remains the standard of care for patients with ELM.

Acknowledgements We thank all of the patients, their families, the investigators and medical staff members who participated in this study. The authors also thank M. Miyazawa (Japan Clinical Oncology Group Data Center) for assistance and support with data collection, and $\mathrm{H}$. Fukuda (Japan Clinical Oncology Group Data Center) for oversight of the study.

Funding This study was supported by the National Cancer Center Research and Development Fund (23-A-16, 23-A-19, 26-A-4, 29-A-3) and a Health and Labour Sciences Research Grant for Clinical Cancer Research (H22-Gan-016) from the Ministry of Health, Labour and Welfare, Japan.

\section{Compliance with ethical standards}

Conflicts of interest Daisuke Takahari reports grants and personal fees from Taiho Pharmaceutical Co. and Ono Pharmaceutical Co., and personal fees from Eli Lilly Japan, Bristol-Myers Squibb, Yakult Honsha Co., Ltd., Chugai Pharmaceutical Co., Ltd., outside the submitted work. Seiji Ito reports grants from National Cancer Center, Japan, Ministry of Health, Labour and Welfare, Japan, during the conduct of the study, grants and personal fees from Chugai Pharmaceutical Co., Ltd., Ono Pharmaceutical Co., personal fees from Taiho Pharmaceutical Co., grants from Merck Sharp and Dohme, outside the submitted work. Junki Mizusawa reports grants from Ministry of Health, Labour and Welfare, Japan, Japan Agency for Medical Research and Development (AMED), during the conduct of the study. Hiroshi Katayama reports grants from Ministry of Health, Labour and Welfare, Japan, AMED, during the conduct of the study; personal fees from Johnson \& Johnson, outside the submitted work. Masanori Terashima reports personal fees from Taiho Pharmaceutical Co., Chugai Pharmaceutical Co., Ltd., Ono Pharmaceutical Co., Bristol-Myers Squibb, Yakult Honsha Co., Ltd., Takeda Pharmaceutical Company Ltd., Eli Lilly Japan, Daiichi Sankyo, outside the submitted work. Mitsuru Sasako reports grants from Japanese Government, personal fees from Taiho Pharmaceutical Co, Sanofi Aventis, during the conduct of the study; grants from Japanese Goverment, personal fees from Chugai Pharmaceutical Co., Taiho Pharmaceutical Co., Yakult Honsha Co., Ltd., Sanofi Aventis, Eli Lilly Japan, Merck Sharp and Dohme Japan, outside 
the submitted work. Takeshi Sano received personal fees from Taiho Pharmaceutical Co. during the conduct of the study and has received personal fees from Chugai Pharmaceutical Co, Chugai Pharmaceutical Co., Ltd., Daiichi Sankyo, Ono Pharmaceutical Co., Eli Lilly Japan, Ethicon, Covidien, Olympus, outside of the submitted work. Shinji Morita, Takashi Nomura, Makoto Yamada, Yoshiyuki Fujiwara, Yutaka Kimura, Atsuki Ikeda, and Yoshio Kadokawa have no conflicts of interest to disclose.

Human rights statement and informed consent All procedures followed were in accordance with the ethical standards of the responsible committee on human experimentation (institutional and national) and with the Helsinki Declaration of 1964 and later versions. Informed consent or an appropriate substitute for it was obtained from all patients for their inclusion in this study.

\section{References}

1. Park I, Kim S, Kim Y, Ryu K, Lee J, Lee J, et al. Clinical characteristics and treatment outcomes of gastric cancer patients with isolated para-aortic lymph node involvement. Cancer Chemother Pharmacol. 2011;67:127-36.

2. Yoshikawa T, Sasako M, Yamamoto S, Sano T, Imamura H, Fujitani K, et al. Phase II study of neoadjuvant chemotherapy and extended surgery for locally advanced gastric cancer. Br J Surg. 2009;96:1015-22.

3. Tsuburaya A, Mizusawa J, Tanaka Y, Fukushima N, Nashimoto A, Sasako M, et al. Neoadjuvant chemotherapy with S-1 and cisplatin followed by D2 gastrectomy with para-aortic lymph node dissection for gastric cancer with extensive lymph node metastasis. $\mathrm{Br}$ J Surg. 2014;101:653-60.

4. Ito S, Sano T, Mizusawa J, Takahari D, Katayama H, Katai $\mathrm{H}$, et al. A phase II study of preoperative chemotherapy with docetaxel, cisplatin, and S-1 followed by gastrectomy with D2 plus para-aortic lymph node dissection for gastric cancer with extensive lymph node metastasis: JCOG1002. Gastric Cancer. 2017;20:322-31.

5. Therasse P, Arbuck S, Eisenhauer E, Wanders J, Kaplan R, Rubinstein L, et al. New guidelines to evaluate the response to treatment in solid tumors. European Organization for Research and Treatment of Cancer, National Cancer Institute of the United States, National Cancer Institute of Canada. J Natl Cancer Inst. 2000;92:205-16.
6. National Cancer Institute. Common Terminology Criteria for Adverse Events (CTCAE) v4.0. https://ctep.cancer.gov/proto colDevelopment/electronic_applications/ctc.htm\#ctc_40. Accessed 9 June 2016.

7. Gastreic J. Japanese classification of gastric carcinoma. Gastric Cancer. 2011;14:101-12.

8. Koizumi W, Nakayama N, Tanabe S, Sasaki T, Higuchi K, Nishimura K, et al. A multicenter phase II study of combined chemotherapy with docetaxel, cisplatin, and S-1 in patients with unresectable or recurrent gastric cancer (KDOG 0601). Cancer Chemother Pharmacol. 2012;69:407-13.

9. Yamada Y, Boku N, Mizusawa J, Iwasa S, Kadowaki S, Nakayama $\mathrm{N}$, et al. Docetaxel plus cisplatin and S-1 versus cisplatin and S-1 in patients with advanced gastric cancer (JCOG1013): an openlabel, phase 3, randomised controlled trial. Lancet Gastroenterol Hepatol. 2019;4:501-10.

10. Al-Batran S-E, Homann N, Pauligk C, Goetze T, Meiler J, Kasper $\mathrm{S}$, et al. Perioperative chemotherapy with fluorouracil plus leucovorin, oxaliplatin, and docetaxel versus fluorouracil or capecitabine plus cisplatin and epirubicin for locally advanced, resectable gastric or gastro-oesophageal junction adenocarcinoma (FLOT4): a randomised, phase 2/3 trial. Lancet. 2019;393:1948-57.

11. Park I, Ryu M, Choi Y, Kang H, Yook J, Park Y, et al. A phase II study of neoadjuvant docetaxel, oxaliplatin, and S-1 (DOS) chemotherapy followed by surgery and adjuvant S-1 chemotherapy in potentially resectable gastric or gastroesophageal junction adenocarcinoma. Cancer Chemother Pharmacol. 2013;72:815-23.

12. Kang Y-K, Yook JH, Ryu M-H, Lee JS, Park Y, Chung I-J, et al. A randomized phase III study of neoadjuvant chemotherapy with docetaxel(D), oxaliplatin(O), and S-1(S) (DOS) followed by surgery and adjuvant S-1 vs. surgery and adjuvant S-1 for resectable advanced gastric cancer (PRODIGY). J Clin Oncol. 2015;33: (suppl; tps4136).

13. Yoshikawa T, Sakamaki K, Nishikawa K, Fujitani K, Tanabe K, Ito $\mathrm{Y}$, et al. Primary results of a randomized two-by-two factorial phase II trial comparing neoadjuvant chemotherapy with two and four courses of cisplatin/S-1 (CS) and docetaxel/cisplatin/S-1 (DCS) as neoadjuvant chemotherapy for locally advanced gastric cancer. J Clin Oncol. 2019;37:(suppl 4; abstr 93).

Publisher's Note Springer Nature remains neutral with regard to jurisdictional claims in published maps and institutional affiliations. 